\title{
Identification of open chromosomal regions and key genes in prostate cancer via integrated analysis of DNase-seq and RNA-seq data
}

\author{
XIN WEI ${ }^{1}$, LILI YU ${ }^{2}$, XUEFEI JIN ${ }^{1}$, LIDE SONG $^{3}$, YANTING LV $^{3}$ and YUPING HAN ${ }^{1}$ \\ Departments of ${ }^{1}$ Urology and ${ }^{2}$ Radiology, China-Japan Union Hospital of Jilin University, Changchun, Jilin 130033; \\ ${ }^{3}$ Department of Pathology, Zhuji People's Hospital, Zhuji, Zhejiang 311800, P.R. China
}

Received July 13, 2017; Accepted January 11, 2018

DOI: $10.3892 / \mathrm{mmr} .2018 .9193$

\begin{abstract}
Prostate cancer is a type of adenocarcinoma arising from the peripheral zone of the prostate gland, and metastasized prostate cancer is incurable with the current available therapies. The present study aimed to identify open chromosomal regions and differentially expressed genes (DEGs) associated with prostate cancer development. The DNase sequencing data (GSE33216) and RNA sequencing data (GSE22260) were downloaded from the Gene Expression Omnibus database. DNase I hypersensitive sites were detected and analyzed. Subsequently, DEGs were identified and their potential functions were enriched. Finally, upstream regulatory elements of DEGs were predicted. In LNCaP cells, following androgen receptor activation, 244 upregulated and 486 downregulated open chromosomal regions were identified. However, only $1 \%$ of the open chromosomal regions were dynamically altered. The 41 genes with upregulated open chromosomal signals within their promoter regions were primarily enriched in biological processes. Additionally, 211 upregulated and 150 downregulated DEGs were identified in prostate cancer, including eight transcription factors (TFs). Finally, nine regulatory elements associated with prostate cancer were predicted. In particular, inhibitor of DNA binding 1 HLH protein (ID1) was the only significantly upregulated TF which exhibited motif enrichment in the promoter regions of upregulated genes. CCCTC-binding factor (CTCF) and ELK1 ETS transcription factor (ELK1), enriched in the open promoter regions of downregulated genes, were potential upstream regulatory elements. Furthermore, reverse transcription-quantitative polymerase chain reaction analysis confirmed that ID1 expression was significantly
\end{abstract}

Correspondence to: Dr Yuping Han, Department of Urology, China-Japan Union Hospital of Jilin University, 126 Xiantai Street, Changchun, Jilin 130033, P.R. China

E-mail: yupingh2017@163.com

Key words: differentially expressed gene, DNase sequencing, open chromosomal region, prostate cancer, regulatory element, RNA sequencing upregulated in LNCaP cells and $5 \alpha$-dihydrotestosterone (DHT)-treated LNCaP cells compared with that in BPH1 cells, while CTCF and ELK1 expression was significantly downregulated in LNCaP cells and DHT-treated LNCaP cells. In conclusion, ID1, CTCF and ELK1 may be associated with prostate cancer, and may be potential therapeutic targets for the treatment of this disease.

\section{Introduction}

Prostate carcinoma is an adenocarcinoma arising from the peripheral zone of the prostate gland and is common in elderly men, with an average onset age of 72 years $(1,2)$. Pathological types of prostate carcinoma include adenocarcinoma, duct adenocarcinoma, urothelial cancer, epidermoid carcinoma and adenosquamous carcinoma (3). The growth rate of prostate carcinoma is variable (4). Metastasized prostate cancer is incurable with the current therapies, and median survival is generally 1-3 years (5). A deep understanding of the molecular mechanism underlying prostate carcinoma development may facilitate the design of novel strategies for the treatment of this disease.

The development and growth of prostate cancer depends on the androgen receptor (AR) protein, which is the intracellular mediator of androgen action. Aberrant activation of AR contributes to the progression of prostate carcinoma to an androgen-independent stage (6). Previous studies have reported that interfering with the synthesis of AR in cancer tissues may control cell differentiation and effectively induce apoptosis in prostate cancer $(7,8)$. Increased AR expression has been demonstrated to be associated with the development of hormone refractory prostate cancer (9). As a transcriptional coactivator of AR, coactivator-associated arginine methyltransferase 1 is upregulated in prostate carcinoma, and it may be a novel therapeutic target in hormone-independent prostate carcinoma (10). Interleukin 6 may promote the progression of androgen-independent prostate cancer by facilitating AR expression and activating AR $(11,12)$. However, the molecular events underlying prostate cancer development are largely unknown.

Genome-wide measurements of protein-DNA interactions and the transcriptome are increasingly being performed 
using deep DNA sequencing methods [chromatin immunoprecipitation sequencing (ChIP-Seq) and RNA sequencing (RNA-seq)] (13). In a previous study, He et al (14) developed and analyzed DNAse sequencing (DNase-seq) dataset GSE33216, and demonstrated that AR and estrogen receptor- $\alpha$ had distinct modes of interaction with chromatin, and that DNase I hypersensitivity dynamics provided a general approach for predicting cell-type specific cistromes. Hu et al (15) identified 320 differentially expressed genes (DEGs) between prostate cancer samples and normal controls by generating RNA-seq dataset GSE22260. However, there has been no integrated analysis of DNase-seq and RNA-seq data, to the best of our knowledge. Therefore, the above DNase-seq and RNA-seq data was downloaded in the present study, and integrated analysis was performed to further examine the open chromosomal regions adjacent to the promoters of the abnormal genes and the corresponding transcriptional regulatory elements that were associated with prostate cancer development. The influences of AR activation on chromosome structure were assessed in prostate carcinoma cells, based on the public DNase-seq data. Potential functions of open chromosomal regions with dynamic alterations were annotated. In addition, combined with the public RNA-seq data, every open chromosomal region adjacent to the promoter of an abnormal gene was detected. Finally, transcriptional regulatory elements crucial to the development of prostate carcinoma were analyzed. The present study may provide fundamental data on the pathogenic mechanisms of prostate carcinoma, and the predicted regulatory elements may be novel targets for the treatment of this disease.

\section{Materials and methods}

Data sources. The DNase-seq data GSE33216 (14) were downloaded from the Gene Expression Omnibus (GEO) database (www.ncbi.nlm.nih.gov/geo) of the National Center for Biotechnology Information (NCBI), including DNase-seq data for LNCaP cells prior to (GSM822387) and following (GSM822388) stimulation with androgen. To generate this dataset, androgen-dependent LNCaP prostate cancer cells were maintained in RPMI-1640 medium containing $10 \%$ fetal bovine serum (FBS). LNCaP cells were starved in phenol-red-free medium containing 10\% charcoal-stripped FBS for 3 days and subsequently treated with $10 \mathrm{nM}$ active androgen $5 \alpha$-dihydrotestosterone (DHT) or ethanol for $4 \mathrm{~h}$ (14). This dataset was sequenced on an Illumina HiSeq2000 system (Illumina, Inc., San Diego, CA, USA) using single-end sequencing.

The RNA-seq data GSE22260 (15) were additionally downloaded from the NCBI GEO database, including four prostate cancer samples (GSM554078, GSM554082, GSM554086 and GSM554088) and four matched normal prostate samples surrounding prostate tumor tissues (GSM554118, GSM554120, GSM554122 and GSM554124). The RNA-seq data were sequenced on the platform Illumina GA II (Illumina, Inc.) using paired-end sequencing.

Data processing. All DNase-seq data were mapped to the reference human genome (hg19) of the University of California, Santa Cruz (genome.ucsc.edu) using Bowtie 0.12.9 (16).
Each read had a unique align position and no more than two mismatches. Other parameters were the default settings. Reads that met the above conditions were extracted.

All RNA-seq data were aligned using Tophat software (version 1.3.1) (17). For each read with a unique align position, mismatches of $\leq 2$ bases were permitted. Other parameters were set to the default. Following read alignment, based on the gene annotation data of the Reference Sequence database (www.ncbi.nlm.nih.gov/RefSeq), transcripts were assembled using Cufflinks (version 1.0.3) (18) and their expression levels were calculated using the fragments per kilobase per million reads method in Cuffdiff (version 2.1.1) (18).

Genome-wide detection of DNase I hypersensitive sites $(D H S)$. All polymerase chain reaction (PCR) duplicates were removed using SAMtools (version 0.1.19) (19). For multireads aligned with the same chromosome site, truncation was performed and only one read was allowed in one align position. Subsequently, peak calling was performed on processed reads via Model-based Analysis of ChIP-Seq (MACS 1.4.0) (20). A P-value $<10^{-5}$ was set as the cut-off criterion.

Dynamically altered DHS among samples. All detected DHS were integrated. Subsequently, reads enriched in peak regions were normalized using the random particle-mesh method (21), to eliminate the bias caused by the length of the peak region and the differences in available reads numbers. The difference of peak enrichment between cancer and normal samples was analyzed using NOISeq (version 1.10) (22) with a q-value $\geq 0.8$.

DEG identification. DEGs between cancer and normal samples were identified via paired t-tests (23). Genes with $\log _{2}$ fold change (FC) $\mid \geq 2$ and a P-value $<0.05$ were considered to be differentially expressed.

Functional enrichment analysis and annotation of specific genes. Gene Ontology (GO) functional enrichment analysis of DEGs was performed using the Database for Annotation, Visualization and Integrated Discovery (DAVID) (24). Subsequently, based on the database of transcription factors (TFs), DEGs with regulatory functions were selected and annotated. Finally, according to the tumor suppressor genes (25) and Disease and Gene Annotation (dga.nubic.northwestern. edu) databases, oncogenes and tumor suppressor genes were selected from the DEGs.

Detection of upstream regulatory element of DEGs. In the present study, the promoter region was from $1 \mathrm{~kb}$ upstream to $0.5 \mathrm{~kb}$ downstream of the transcription start site (TSS). For the promoter regions of upegulated and downregulated genes, motif finding was performed using Seqpos (version 1.0.0) (26) to predict the TFs of DEGs. A P-value $<10^{-5}$ was set as the cut-off criterion.

Reverse transcription-quantitative PCR (RT-qPCR). To verify the results of the analyses described above, the expression levels of key transcriptional regulatory elements in human LNCaP cells were detected by RT-qPCR. In the present study, untransformed prostate epithelial BPH-1 cells and androgen-dependent LNCaP cells were obtained from the 
A

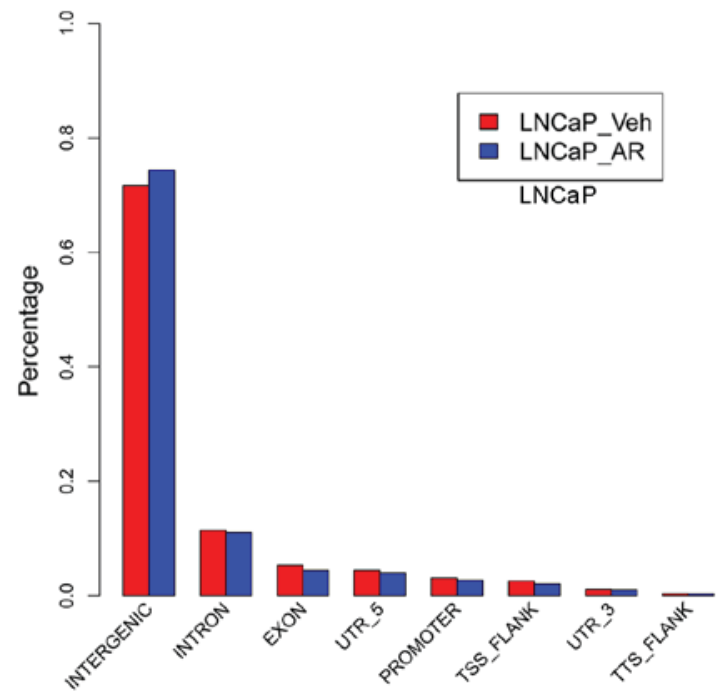

B

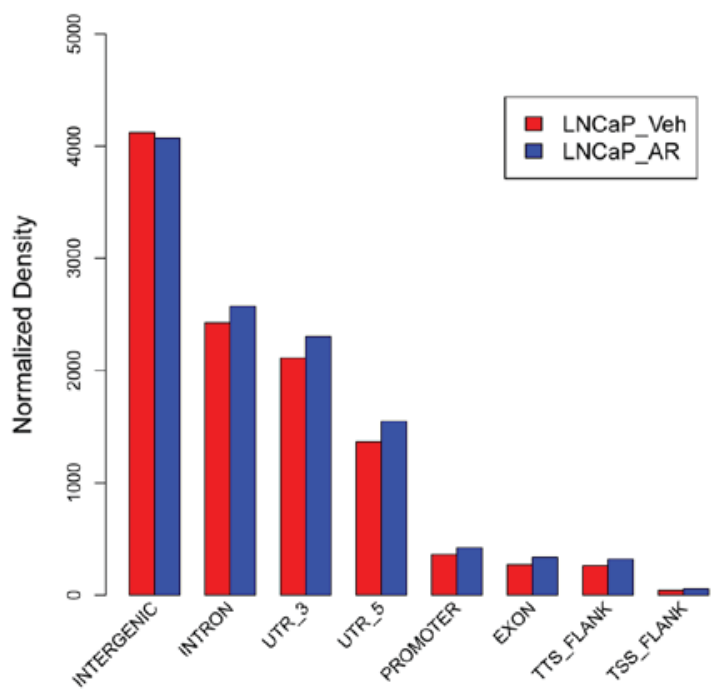

Figure 1. Distribution of open genomic regions in the LNCaP cell line prior to (red columns) and following (blue columns) androgen stimulation. (A) Open genomic region count. (B) Density distribution of the open genomic region. AR, androgen receptor; Veh, vehicle; UTR, untranslated region; TTS, transcription termination site; TSS, transcription start site.

American Type Culture Collection (Manassas, VA, USA). As previously described (14), the LNCaP cells were starved in phenol-red-free medium (Gibco; Thermo Fisher Scientific, Inc.) containing 10\% charcoal-stripped FBS (Gibco; Thermo Fisher Scientific, Inc.) and maintained in a $37^{\circ} \mathrm{C}$ incubator with $5 \% \mathrm{CO}_{2}$ for 3 days, followed by treatment with $10 \mathrm{nM}$ DHT for $4 \mathrm{~h}$. BPH-1 cells were starved for 3 days as the control group. Therefore, three groups were obtained, including BPH-1, $\mathrm{LNCaP}$, and LNCaP + DHT. Total RNA was isolated from the different treated groups using TRIzol reagent (Invitrogen; Thermo Fisher Scientific, Inc., Waltham, MA, USA), according to the manufacturer's protocol. Following measurement of the concentration of total RNA with a NanoDrop 2000 spectrometer (Thermo Fisher Scientific, Inc., Wilmington, DE, USA), RT was performed using the PrimeScript RT Master Mix kit (Takara Bio, Inc., Otsu, Japan). Subsequently, qPCR was performed using a SYBR-Green kit (Applied Biosystems; Thermo Fisher Scientific, Inc.), in accordance with the manufacturer's protocol. The primer sequences $\left(5^{\prime}-3^{\prime}\right)$ of the selected DEGs were used as follows: inhibitor of DNA binding $1 \mathrm{HLH}$ protein (ID1), forward CTGCTCTACGACATGAACGG and reverse GAAGGTCCCTGATGTAGTCGAT; CCCTC-binding factor (CTCF), forward CAGTGGAGAATTGGTTCGGCA and reverse CTGGCGTAATCGCACATGGA; ELK1 ETS transcription factor (ELK1) forward CCCGTCCGTGGCCTT ATTTA and reverse CTCTGCATCCACCAGCTTGA; and GAPDH, forward TGACAACTTTGGTATCGTGGAAGG and reverse AGGCAGGGATGATGTTCTGGAGAG. The thermocycling conditions for the qPCR were set as follows: $50^{\circ} \mathrm{C}$ for $3 \mathrm{~min}, 95^{\circ} \mathrm{C}$ for $3 \mathrm{~min}, 40$ cycles of $95^{\circ} \mathrm{C}$ for $10 \mathrm{sec}$ and $60^{\circ} \mathrm{C}$ for $30 \mathrm{sec}$, and a final melt curve $\left(60-95^{\circ} \mathrm{C}\right.$ with $0.5^{\circ} \mathrm{C}$ increments for $10 \mathrm{sec}$ ). The expression levels of selected DEGs were normalized to GAPDH and calculated using the $2^{-\Delta \Delta C q}$ method, as described previously (27). Each sample was analyzed in triplicate. The obtained data are presented as the mean \pm standard deviation. Significant differences between groups were analyzed using the post hoc Tukey test following one-way analysis of variance, using SPSS 22.0 software (IBM Corp., Armonk, NY, USA). The results were visualized using GraphPad Prism 5.0 software (GraphPad Software, Inc., La Jolla, CA, USA). P $<0.05$ was considered to indicate a statistically significant difference.

\section{Results}

Distribution of the open chromosomal regions in LNCaP cells. In the LNCaP cells prior to and following androgen stimulation, the number and distribution of open chromosomal regions in different functional genomic regions were analyzed. As presented in Fig. 1A, in LNCaP cells prior to androgen stimulation, there were 69,044 open chromosomal regions with an overall length of 94,915,563 bp and an average length of $1,375 \mathrm{bp}$, accounting for $\sim 3.16 \%$ of the total chromosomal regions; following androgen stimulation, there were 70,141 open chromosomal regions with a total length of $88,852,553 \mathrm{bp}$ and an average length of $1,267 \mathrm{bp}$, accounting for $~ 2.96 \%$ of the overall chromosomal regions. However, androgen stimulation exerted no influence on the gene distribution of the open chromosomal regions (Fig. 1B), and the majority of the open chromosomal regions were located in intergenic regions, followed by introns, exons, 5 ' untranslated regions, promoter regions, and the surrounding regions of transcription termination sites (TTS) and TSS.

Detection of DHS regions and functional enrichment analysis. In order to detect whether open chromosomal regions in LNCaP cells altered dynamically following the activation of AR induced by androgen, the different dysregulated open chromosomal regions in $\mathrm{LNCaP}$ cells prior to and following androgen stimulation were analyzed using a permutation test. A total of 244 upregulated and 486 downregulated open chromosomal regions were identified in $\mathrm{LNCaP}$ cells following $\mathrm{AR}$ 
Table I. Enriched functions for genes adjacent to the open chromosomal regions.

\begin{tabular}{|c|c|c|c|}
\hline Term & No.genes & Gene symbols & P-value \\
\hline GO:0006915 apoptosis & 6 & $\begin{array}{l}\text { SGK1, MAP3K5, CDK11A, SOS2, } \\
\text { BUB1B, CDK11B, LOC100133692, KALRN }\end{array}$ & 0.005187071 \\
\hline GO:0006468 protein amino acid phosphorylation & 6 & $\begin{array}{l}\text { SGK1, MAP3K5, CDK11A, NTRK2, } \\
\text { CDK11B, MAPK10, LOC100133692, KALRN }\end{array}$ & 0.007965814 \\
\hline $\begin{array}{l}\text { GO:0008624 induction of apoptosis by } \\
\text { extracellular signals }\end{array}$ & 3 & MAP3K5, SOS2, KALRN & 0.019397183 \\
\hline GO:0050804 regulation of synaptic transmission & 3 & NTRK2, GRIA4, CALB1 & 0.027853425 \\
\hline GO:0007242 intracellular signaling cascade & 7 & $\begin{array}{l}\text { DUSP4, MAP3K5, SOS2, BUB1B, } \\
\text { AKAP7, MAPK10, KALRN }\end{array}$ & 0.028874403 \\
\hline $\begin{array}{l}\text { GO:0051966 regulation of synaptic } \\
\text { transmission, glutamatergic }\end{array}$ & 2 & NTRK2, GRIA4 & 0.03591552 \\
\hline GO:0000165 MAPKKK cascade & 3 & DUSP4, MAP3K5, MAPK10 & 0.048303904 \\
\hline
\end{tabular}

GO, Gene Ontology.

activation. Subsequently, the regions with dynamic alterations were further analyzed to identify their correlations with gene expression. In total, 730 adjacent genes to the open chromosomal regions were examined. The promoters of 41 genes contained upregulated open chromosomal signals and the promoters of 24 genes overlapped with downregulated open chromosomal signals (data not shown). However, only $\sim 1 \%$ of open chromosomal regions changed dynamically.

GO functional enrichment analyses of genes adjacent to the open chromosomal regions were performed. The results demonstrated that 41 genes with upregulated open chromosomal signals within their promoter regions were significantly involved the biological processes of 'apoptosis' $(\mathrm{P}=0.005187071)$, 'protein phosphorylation' $(\mathrm{P}=0.007965814)$, 'regulation of synaptic transmission' $(\mathrm{P}=0.027853425)$ and 'MAPK kinase cascade' $(\mathrm{P}=0.048303904)$ (Table I). Nevertheless, 24 genes with downregulated open chromosomal signals within promoter regions were not significantly enriched in any GO terms.

Identification of crucial open chromosomal regions. Firstly, RNA-seq data (GSE22260) of prostate cancer patients were used to investigate the association between gene expression and the dynamic alterations in open chromosomal regions prior to and following AR activation. In total, 211 upregulated and 150 downregulated genes were identified in prostate cancer samples compared with normal samples. Among the DEGs, eight DEGs served as transcription factors (Table II).

Subsequently, the correlation analysis between dynamic alterations in open chromosomal regions and gene expression regulation was performed. However, only one of the 226 open chromosomal regions adjacent to downregulated genes exhibited a downregulated signal following the activation of AR, which was in accordance with that of the 243 open chromosomal regions adjacent to the upregulated genes (Table III).

Motif analysis of transcription factors in crucial open chromosomal regions. Based on dynamic alterations in open chromosomal regions and the screened DEGs, TF motif
Table II. Identification of differentially expressed genes and $\mathrm{TF}$ in prostate cancer.

\begin{tabular}{|c|c|c|c|}
\hline & $\begin{array}{l}\text { No. } \\
\text { genes }\end{array}$ & $\begin{array}{l}\text { No. } \\
\text { TF }\end{array}$ & TF symbols \\
\hline Downregulated & 211 & 4 & $\begin{array}{l}\text { IFI16, NEUROG3, RARG, } \\
\text { SIM1 }\end{array}$ \\
\hline Upregulated & 150 & 4 & $\begin{array}{l}\text { DMBX1,NCOA2, ONECUT2, } \\
\text { ZNF83 }\end{array}$ \\
\hline
\end{tabular}

$\mathrm{TF}$, transcription factors.

scanning was performed. A total of nine regulatory elements associated with DEGs in prostate cancer were identified, including CTCF, ELK1 and zinc finger protein 143 (ZNF143) enriched in open promoter regions of downregulated DEGs, and cone-rod homeobox (CRX), GA binding protein transcription factor $\alpha$ subunit (GABPA), histone $\mathrm{H} 4$ transcription factor (HINFP), ID1, methyl-CpG binding protein 2 (MECP2) and paired box 6 (PAX6) enriched in open promoter regions of upregulated genes (Table IV). Notably, ID1 was the only significantly upregulated TF that exhibited motif enrichment in promoter regions of upregulated genes (Fig. 2).

Validation of gene expression using RT-qPCR. In order to validate the results of the above bioinformatics analyses, the expression levels of key transcriptional regulatory elements, including ID1, CTCF and ELK1, were detected by RT-qPCR. ID1 was the only significantly upregulated TF which exhibited motif enrichment in the promoter regions of upregulated genes. CTCF and ELK1 were potential upstream regulatory elements in the open promoter regions of downregulated DEGs. Consistent with the results of above bioinformatics analyses, the expression of ID1 was significantly upregulated 
Table III. DHS adjacent to upregulated and downregulated genes in prostate cancer.

\begin{tabular}{lccc}
\hline & $\begin{array}{c}\text { DHS upregulated in } \\
\text { AR activation }\end{array}$ & $\begin{array}{c}\text { DHS downregulated in } \\
\text { AR activation }\end{array}$ & $\begin{array}{c}\text { Non-dynamic } \\
\text { DHS }\end{array}$ \\
\hline DHS adjacent to downregulated genes & 1 & 0 & 225 \\
DHS adjacent to upregulated genes & 0 & 0 & 243 \\
\hline
\end{tabular}

AR, androgen receptor; DHS, DNase I hypersensitive sites.

Table IV. Prediction of upstream regulatory elements of differentially expressed genes in prostate cancer.

\begin{tabular}{|c|c|c|c|}
\hline Term & No. genes & Gene symbols & P-value \\
\hline GO:0006915 apoptosis & 6 & $\begin{array}{l}\text { SGK1, MAP3K5, CDK11A, SOS2, } \\
\text { BUB1B, CDK11B, LOC100133692, } \\
\text { KALRN }\end{array}$ & 0.005187071 \\
\hline GO:0006468 protein amino acid phosphorylation & 6 & $\begin{array}{l}\text { SGK1, MAP3K5, CDK11A, NTRK2, } \\
\text { CDK11B, MAPK10, LOC100133692, } \\
\text { KALRN }\end{array}$ & 0.007965814 \\
\hline $\begin{array}{l}\text { GO:0008624 induction of apoptosis by } \\
\text { extracellular signals }\end{array}$ & 3 & MAP3K5, SOS2, KALRN & 0.019397183 \\
\hline GO:0050804 regulation of synaptic transmission & 3 & NTRK2, GRIA4, CALB1 & 0.027853425 \\
\hline GO:0007242 intracellular signaling cascade & 7 & $\begin{array}{l}\text { DUSP4, MAP3K5, SOS2, BUB1B, } \\
\text { AKAP7, MAPK10, KALRN }\end{array}$ & 0.028874403 \\
\hline $\begin{array}{l}\text { GO:0051966 regulation of synaptic transmission, } \\
\text { glutamatergic }\end{array}$ & 2 & NTRK2, GRIA4 & 0.03591552 \\
\hline GO:0000165 MAPKKK cascade & 3 & DUSP4, MAP3K5, MAPK10 & 0.048303904 \\
\hline
\end{tabular}

GO, Gene Ontology.

A

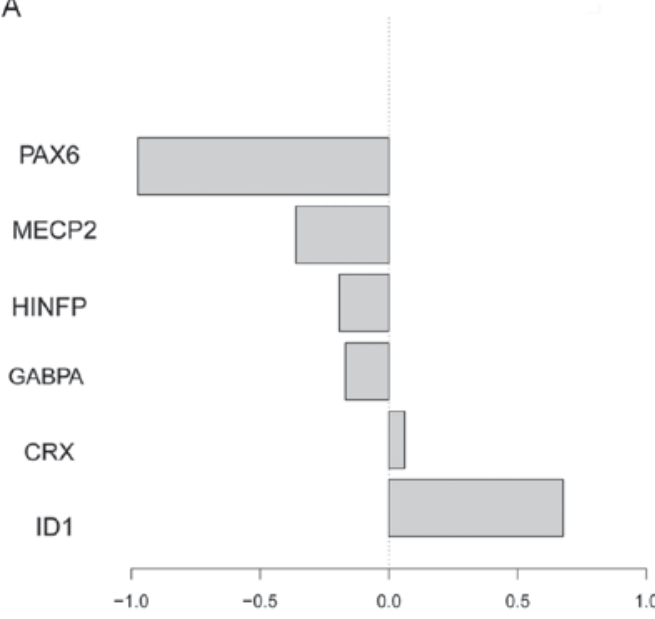

$\log 2$ ratio Prostate cancer vs. Adjacent normal tissue
B

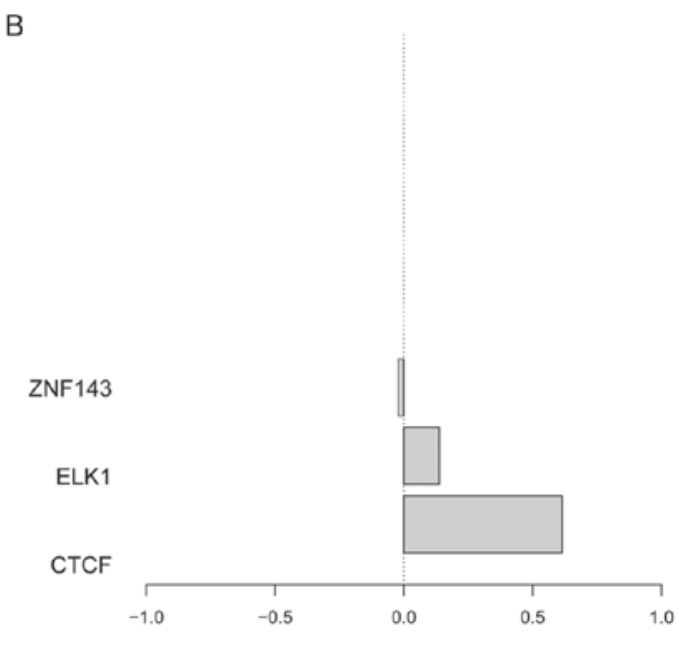

$\log 2$ ratio Prostate cancer vs. Adjacent normal tissue

Figure 2. Expression alterations in upstream regulatory elements in the abnormal genes. (A) TF motif from DHS adjacent to upregulated genes. (B) TF motif from DHS adjacent to downregulated genes. TF, transcription factor; DHS, DNase I hypersensitive sites; PAX6, paired box 6; MECP2, methyl-CpG binding protein 2; HINFP, histone H4 transcription factor; GABPA, GA binding protein transcription factor $\alpha$ subunit; CRX, cone-rod homeobox; ID1, inhibitor of DNA binding $1 \mathrm{HLH}$ protein; ZNF143, zinc finger protein 143; ELK1, ELK1 ETS transcription factor; CTCF, CCCTC-binding factor.

in LNCaP cells and DHT-treated LNCaP cells compared with that in BPH1 cells $(\mathrm{P}<0.05$; Fig. 3A), whereas CTCF and
ELK1 were significantly downregulated in LNCaP cells and DHT-treated LNCaP cells ( $\mathrm{P}<0.05$; Fig. $3 \mathrm{~B}$ and $\mathrm{C}$ ). 
A

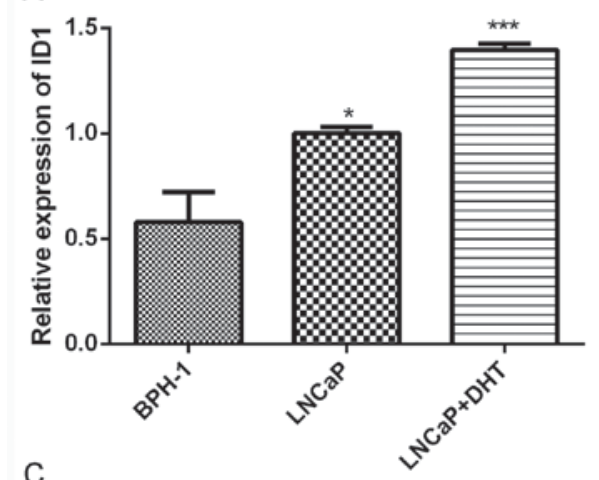

C

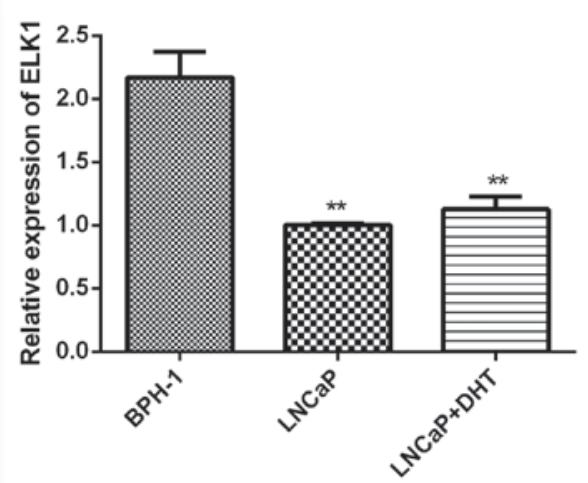

B

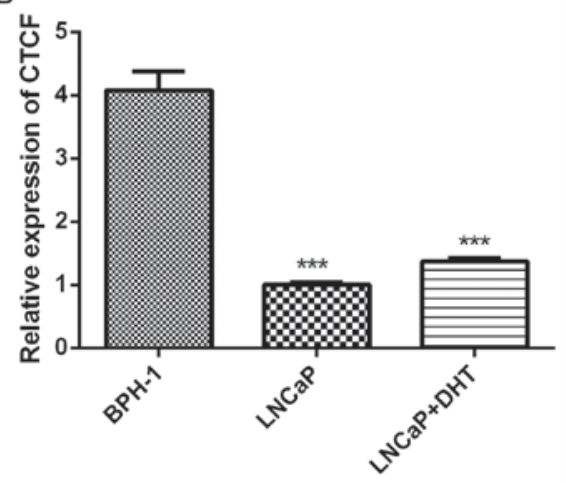

Figure 3. Expression levels of (A) ID1, (B) CTCF and (C) ELK1 transcriptional regulatory elements in BPH1, LNCaP and DHT-treated LNCaP cells. Data are presented as the mean \pm standard error of the mean. ${ }^{*} \mathrm{P}<0.05,{ }^{* *} \mathrm{P}<0.01$ and ${ }^{* * * *} \mathrm{P}<0.001$ vs. BPH-1 cell group. DHT, $5 \alpha$-dihydrotestosterone; ELK1, ELK1 ETS transcription factor; CTCF, CCCTC-binding factor; ID1, inhibitor of DNA binding 1 HLH protein.

\section{Discussion}

Previous studies have indicated that prostate cancer development is associated with genetic and epigenetic alterations induced by AR activation $(28,29)$. The application of cytogenetic and molecular genetic methods has led to the identification of numerous tumor-associated chromosomal regions during the tumorigenesis of prostate cancer (30). In present study, 244 upregulated and 486 downregulated open chromosomal regions were identified in $\mathrm{LNCaP}$ cells following AR activation induced by androgen. However, only $\sim 1 \%$ of open chromosomal regions altered dynamically following AR activation, indicating the limited influence of the activated AR on dynamic alterations in the chromosome. In addition, 211 upregulated and 150 downregulated DEGs were identified in prostate cancer samples compared with normal samples. There was only one, out of the 226 open chromosomal regions adjacent to downregulated genes, which exhibited a downregulated signal post-AR activation, which was in accordance with that of the 243 open chromosomal regions adjacent to upregulated genes. These results suggested that dynamic alterations of open chromosomal regions following AR activation did not affect gene expression regulation in prostate cancer. Notably, nine regulatory elements (CTCF, ELK1, ZNF143, CRX, GABPA, HINFP, ID1, MECP2 and PAX6) of DEGs were identified. ID1 was the only significantly upregulated TF which exhibited motif enrichment in the promoter regions of upregulated genes. CTCF and ELK1 were potential upstream regulatory elements. Notably, consistent with the results of the above bioinformatics analyses, RT-qPCR analysis confirmed that ID1 was significantly upregulated in LNCaP cells and DHT-treated LNCaP cells compared with that in BPH1 cells, whereas CTCF and ELK1 were significantly downregulated in LNCaP cells and DHT-treated LNCaP cells.

ID1 is a member of the helix-loop-helix transcription factor family that lacks a basic domain (31). Increased ID1 expression has been confirmed to be associated with cell proliferation, immortalization, invasion and an aggressive malignant phenotype in a number of human cell lines (32). ID1 expression has been detected in numerous types of human cancer (including breast cancer, hepatocellular carcinoma, human oral squamous cell carcinoma, papillary thyroid cancer and pancreatic cancer) and its expression level has been suggested to be a marker for malignant progression in a number of types of human cancer, including prostate cancer $(33,34)$. Furthermore, it has been reported that ID1 may be activated by AR (35). In androgen-dependent prostate cancer, androgen negatively regulates ID1, which may partially induce androgen-independent prostate cancer following prolonged androgen deprivation therapy (36). ID1 may serve as an upstream regulator of nuclear factor- $\mathrm{\kappa} \mathrm{B}$, which may inhibit cellular apoptosis and induce cell proliferation; therefore, the inactivation of ID1 may be a promising therapeutic strategy for promoting chemotherapeutic drug-induced apoptosis in prostate cancer cells (37). Based on the results of the present study, it may be hypothesized that ID1 may have a close association with prostate cancer development.

ELK1, a known carcinogenic factor, is able to stimulate transcription from the c-fos serum response element or from an ETS binding site (38). Transient transfection assays have demonstrated that the androgen receptor-mediated 
activation of mitogen-activated protein kinase results in enhanced activity of the transcription factor ELK1 (39). A previous study reported that ELK1 leads to transcriptional activation by combining with a cis-acting element to promote the expression of downstream genes (40). ELK1 regulates selective and sustained genes that are essential for growth signaling by AR in prostate cancer cells, and the ELK1-AR interaction may be a potential drug target in the treatment of prostate cancer (41). Furthermore, haploinsufficiency of CTCF has been demonstrated to destabilize DNA methylation and to predispose to cancer (42). CTCF has been observed to serve a role in certain human tumors $(43,44)$. Histone methylation and DNA methylation analysis of the imprinting control region of insulin-like growth factor $2 / \mathrm{H} 19$, located at the CTCF binding domain, has increased the understanding of carcinogenesis and may improve the diagnosis of prostate cancer (45). Therefore, CTCF and ELK1 expression levels may be associated with prostate cancer development.

In conclusion, open chromosomal regions adjacent to the promoter of the abnormal genes were analyzed, and the corresponding transcriptional regulatory elements were predicted. In LNCaP cells following AR activation, 244 upregulated and 486 downregulated open chromosomal regions were identified. Furthermore, a total of 211 upregulated and 150 downregulated genes were identified in prostate cancer samples. In addition, ID1, CTCF, and ELK1 may serve a role in prostate cancer development. These results may improve our understanding of prostate carcinoma and provide potential diagnostic targets for treatment. However, the sample size of the present study was small. Furthermore, the RNA-seq data of prostate cancer samples, and not the RNA-seq data for LNCaP cells treated with or without androgen, were analyzed in the present study. If the RNA-seq data for $\mathrm{LNCaP}$ cells treated with or without androgen were additionally analyzed, more direct and notable findings may be obtained for the clearer elucidation of the molecular mechanism underlying prostate cancer development associated with AR activation. Furthermore, the present study did not verify the difference in a large number of cell lines and in human carcinoma tissues. Therefore, further studies with a larger sample size and more datasets are required to confirm the results of the present study.

\section{Acknowledgements}

Not applicable.

\section{Funding}

No funding was received.

\section{Availability of data and materials}

The datasets used and analysed during the current study are available from the corresponding author on reasonable request.

\section{Authors' contributions}

$\mathrm{XJ}$ and LS were involved in the conception and design of the research. YL and YH participated in the acquisition of data. LY and YH performed the analysis and interpretation of data.
XW and LY were involved in the conception of the study, participated in its design and coordination and aided in the writing of the manuscript. All authors read and approved the final manuscript.

\section{Ethics approval and consent to participate}

Not applicable.

\section{Consent for publication}

Not applicable.

\section{Competing interests}

The authors declare that they have no competing interests.

\section{References}

1. Heidenreich A, Bellmunt J, Bolla M, Joniau S, Mason M, Matveev V, Mottet N, Schmid HP, van der Kwast T, Wiegel T, et al: EAU guidelines on prostate cancer. Part 1: Screening, diagnosis, and treatment of clinically localised disease. Eur Urol 59: 61-71, 2011.

2. Howlader N, Noone A, Krapcho M, Neyman N, Aminou R, Waldron W, Altekruse SF, Kosary CL, Ruhl J, Tatalovich Z (eds), et al: SEER cancer statistics review, 1975-2008. Bethesda, MD: National Cancer Institute; 2011. Also available online. Last accessed December 1, 2011.

3. Grignon DJ: Unusual subtypes of prostate cancer. Mod Pathol 17: 316-327, 2004.

4. Siegel R, Naishadham D and Jemal A: Cancer statistics, 2012. CA Cancer J Clin 62: 10-29, 2012.

5. Edge SB and Compton CC: The American Joint Committee on Cancer: The 7th edition of the AJCC cancer staging manual and the future of TNM. Ann Surg Oncol 17: 1471-1474, 2010.

6. Culig Z, Hobisch A, Cronauer MV, Radmayr C, Trapman J, Hittmair A, Bartsch $\mathrm{G}$ and Klocker $\mathrm{H}$ : Androgen receptor activation in prostatic tumor cell lines by insulin-like growth factor-I, keratinocyte growth factor, and epidermal growth factor. Cancer Res 54: 5474-5478, 1994.

7. Collins AT, Berry PA, Hyde C, Stower MJ and Maitland NJ: Prospective identification of tumorigenic prostate cancer stem cells. Cancer Res 65: 10946-10951, 2005.

8. Heinlein $\mathrm{CA}$ and Chang $\mathrm{C}$ : Androgen receptor (AR) coregulators: An overview. Endocr Rev 23: 175-200, 2002.

9. Edwards J, Krishna N, Grigor K and Bartlett J: Androgen receptor gene amplification and protein expression in hormone refractory prostate cancer. Br J Cancer 89: 552-556, 2003.

10. Hong H, Kao C, Jeng MH, Eble JN, Koch MO, Gardner TA, Zhang S, Li L, Pan CX, Hu Z, et al: Aberrant expression of CARM1, a transcriptional coactivator of androgen receptor, in the development of prostate carcinoma and androgen-independent status. Cancer 101: 83-89, 2004.

11. Lin DL, Whitney MC, Yao Z and Keller ET: Interleukin-6 induces androgen responsiveness in prostate cancer cells through up-regulation of androgen receptor expression. Clin Cancer Res 7: 1773-1781, 2001.

12. Malinowska K, Neuwirt H, Cavarretta IT, Bektic J, Steiner H, Dietrich H, Moser PL, Fuchs D, Hobisch A and Culig Z: Interleukin-6 stimulation of growth of prostate cancer in vitro and in vivo through activation of the androgen receptor. Endocr Relat Cancer 16: 155-169, 2009.

13. Pepke S, Wold B and Mortazavi A: Computation for ChIP-seq and RNA-seq studies. Nat Methods 6 (11 Suppl): S22-S32, 2009.

14. He HH, Meyer CA, Chen MW, Jordan VC, Brown M and Liu XS: Differential DNase I hypersensitivity reveals factor-dependent chromatin dynamics. Genome Res 22: 1015-1025, 2012.

15. Hu FY, Zhao XM, Tang NL, Zhang Y and Chen L: Comparative analysis of protein-coding genes and long non-coding RNAs of prostate cancer between Caucasian and Chinese populations. In: Systems Biology (ISB), 2012 IEEE 6th International Conference on IEEE, pp291-296, 2012. 
16. Li $\mathrm{H}$ and Durbin R: Fast and accurate long-read alignment with Burrows-Wheeler transform. Bioinformatics 26: 589-595, 2010.

17. Trapnell C, Pachter L and Salzberg SL: TopHat: Discovering splice junctions with RNA-Seq. Bioinformatics 25: 1105-1111, 2009.

18. Trapnell C, Williams BA, Pertea G, Mortazavi A, Kwan G, van Baren MJ, Salzberg SL, Wold BJ and Pachter L: Transcript assembly and quantification by RNA-Seq reveals unannotated transcripts and isoform switching during cell differentiation. Nat Biotechnol 28: 511-515, 2010

19. Li H, Handsaker B, Wysoker A, Fennell T, Ruan J, Homer N, Marth G, Abecasis G and Durbin R; 1000 Genome Project Data Processing Subgroup: The sequence alignment/map format and SAMtools. Bioinformatics 25: 2078-2079, 2009

20. Zhang Y, Liu T, Meyer CA, Eeckhoute J, Johnson DS, Bernstein BE, Nusbaum C, Myers RM, Brown M, Li W and Liu XS: Model-based analysis of ChIP-Seq (MACS). Genome Biol 9: R137, 2008.

21. Ewert R: Broadband slat noise prediction based on CAA and stochastic sound sources from a fast random particle-mesh (RPM) method. Comput Fluids 37: 369-387, 2008.

22. Tarazona S, García-Alcalde F, Dopazo J, Ferrer A and Conesa A: Differential expression in RNA-seq: A matter of depth. Genome Res 21: 2213-2223, 2011.

23. Hsu $\mathrm{H}$ and Lachenbruch PA: Paired t test. Wiley Encyclopedia of Clinical Trials, 2008.

24. Huang da W, Sherman BT and Lempicki RA: Systematic and integrative analysis of large gene lists using DAVID bioinformatics resources. Nat Protoc 4: 44-57, 2009.

25. Zhao M, Sun J and Zhao Z: TSGene: A web resource for tumor suppressor genes. Nucleic Acids Res 41: D970-D976, 2013.

26. He HH, Meyer CA, Shin H, Bailey ST, Wei G, Wang Q, Zhang Y, $\mathrm{Xu} \mathrm{K}, \mathrm{Ni}$ M, Lupien M, et al: Nucleosome dynamics define transcriptional enhancers. Nat Genet 42: 343-347, 2010.

27. Livak KJ and Schmittgen TD: Analysis of relative gene expression data using real-time quantitative PCR and the $2^{-\Delta \Delta C_{\mathrm{T}}}$ method. Methods 25: 402-408, 2001.

28. Schwarzenbach H, Chun FK, Isbarn H, Huland H and Pantel K: Genomic profiling of cell-free DNA in blood and bone marrow of prostate cancer patients. J Cancer Res Clin Oncol 137: 811-819, 2011.

29. Steinkamp MP, O'Mahony OA, Brogley M, Rehman H, Lapensee EW, Dhanasekaran S, Hofer MD, Kuefer R, Chinnaiyan A, Rubin MA, et al: Treatment-dependent androgen receptor mutations in prostate cancer exploit multiple mechanisms to evade therapy. Cancer Res 69: 4434-4442, 2009.

30. Costa VL, Henrique R and Jerónimo C: Epigenetic markers for molecular detection of prostate cancer. Dis Markers 23:31-41, 2007

31. Perk J, Iavarone A and Benezra R: Id family of helix-loop-helix proteins in cancer. Nat Rev Cancer 5: 603-614, 2005.

32. Parrinello S, Lin CQ, Murata K, Itahana Y, Singh J, Krtolica A, Campisi J and Desprez PY: Id-1, ITF-2, and Id-2 comprise a network of helix-loop-helix proteins that regulate mammary epithelial cell proliferation, differentiation, and apoptosis. J Biol Chem 276: 39213-39219, 2001.
33. Kebebew E, Treseler PA, Duh QY and Clark OH: The helix-loop-helix transcription factor, Id-1, is overexpressed in medullary thyroid cancer. Surgery 128: 952-957, 2000.

34. O'Toole PJ, Inoue T, Emerson L, Morrison IE, Mackie AR, Cherry RJ and Norton JD: Id proteins negatively regulate basic helix-loop-helix transcription factor function by disrupting subnuclear compartmentalization. J Biol Chem 278: 45770-45776, 2003.

35. Ao J, Meng J, Zhu L, Nie H, Yang C, Li J, Gu J, Lin Q, Long W, Dong $\mathrm{X}$ and Li C: Activation of androgen receptor induces ID1 and promotes hepatocellular carcinoma cell migration and invasion. Mol Onco 6: 507-515, 2012.

36. Xu B, Sun Y, Tang G, Xu C, Wang L, Zhang Y and Ji J: Id-1 expression in androgen-dependent prostate cancer is negatively regulated by androgen through androgen receptor. Cancer Lett 278: 220-229, 2009.

37. Ling MT, Wang X, Ouyang XS, Xu K, Tsao SW and Wong YC: Id-1 expression promotes cell survival through activation of NF-kappaB signalling pathway in prostate cancer cells. Oncogene 22: 4498-4508, 2003.

38. Li QJ, Yang SH, Maeda Y, Sladek FM, Sharrocks AD and Martins-Green M: MAP kinase phosphorylation-dependent activation of Elk-1 leads to activation of the co-activator p300. EMBO J 22: 281-291, 2003.

39. Chen A, Xu J and Johnson A: Curcumin inhibits human colon cancer cell growth by suppressing gene expression of epidermal growth factor receptor through reducing the activity of the transcription factor Egr-1. Oncogene 25: 278-287, 2005.

40. Liu T, Wu J and He F: Evolution of cis-acting elements in 5' flanking regions of vertebrate actin genes. J Mol Evol 50: 22-30, 2000.

41. Patki M, Chari V, Sivakumaran S, Gonit M, Trumbly R and Ratnam M: The ETS domain transcription factor ELK1 directs a critical component of growth signaling by the androgen receptor in prostate cancer cells. J Biol Chem 288: 11047-11065, 2013.

42. Kemp CJ, Moore JM, Moser R, Bernard B, Teater M, Smith LE, Rabaia NA, Gurley KE, Guinney J, Busch SE, et al: CTCF haploinsufficiency destabilizes DNA methylation and predisposes to cancer. Cell Reports 7: 1020-1029, 2014.

43. Ulaner GA, Vu TH, Li T, Hu JF, Yao XM, Yang Y, Gorlick R, Meyers P, Healey J, Ladanyi M and Hoffman AR: Loss of imprinting of IGF2 and H19 in osteosarcoma is accompanied by reciprocal methylation changes of a CTCF-binding site. Hum Mol Genet 12: 535-549, 2003

44. Rakha EA, Pinder SE, Paish CE and Ellis IO: Expression of the transcription factor CTCF in invasive breast cancer: A candidate gene located at 16q22.1. Br J Cancer 91: 1591-1596, 2004.

45. Paradowska A, Fenic I, Konrad L, Sturm K, Wagenlehner F, Weidner $\mathrm{W}$ and Steger K: Aberrant epigenetic modifications in the CTCF binding domain of the IGF2/H19 gene in prostate cancer compared with benign prostate hyperplasia. Int $\mathrm{J}$ Oncol 35: 87-96, 2009. 